\title{
Influence of Nationality on the Brief International Cognitive Assessment for Multiple
}

\section{Sclerosis (BICAMS)}

\author{
A Smerbeck ${ }^{1}$, RHB Benedict ${ }^{2}$, A Eshaghi ${ }^{3}$, S Vanotti ${ }^{4}$, CT Spedo ${ }^{5}$, JB Dusankova ${ }^{6}$, MA Sahraian $^{3}$, VD \\ Marques $^{5}$, and DW Langdon ${ }^{7}$ \\ ${ }^{1}$ Department of Psychology, Rochester Institute of Technology, Rochester, NY, USA \\ ${ }^{2}$ Department of Neurology, University at Buffalo, State University of New York (SUNY), Buffalo, NY, USA. \\ ${ }^{3}$ MS Research Center, Neuroscience Institute , Tehran University of Medical Sciences, Tehran, Iran. \\ ${ }^{4}$ Multiple Sclerosis Clinic, INEBA- Neurosciencies Institute of Buenos Aires. Buenos Aires, Argentina. \\ ${ }^{5}$ Department of Neuroscience and Behavior Sciences, Ribeirão Preto Medical School, University of São \\ Paulo (FMRP-USP) - Ribeirão Preto, SP, Brazil. \\ ${ }^{6}$ Department of Neurology and Center of Clinical Neuroscience, Charles University in Prague, 1st Faculty \\ of Medicine and General University Hospital in Prague, Prague, Czech Republic. \\ ${ }^{7}$ Royal Holloway, University of London, Egham, Surrey, UK
}

RUNNING HEAD: Nationality and Regression Based Norms in MS

\section{CORRESPONDENCE:}

A. Smerbeck, PhD

Dept. of Psychology, Rochester Institute of Technology, 1 Lomb Memorial Drive, Rochester, NY 14203 Telephone: +1-585-475-3593

Email: amsgss@rit.edu 


\section{$\underline{\text { Abstract }}$}

Objective: In answer to the call for improved accessibility of neuropsychological services to the international community, the Brief International Cognitive Assessment for Multiple Sclerosis (BICAMS; MS) was validated in multiple, non-English speaking countries. It was created to monitor processing speed and learning in MS patients, including abbreviated versions of the Symbol Digit Modalities Test (SDMT), California Verbal Learning Test, 2nd Edition (CVLT2), and the Brief Visuospatial Memory Test, Revised (BVMTR). The objective of the present study was to examine whether participant nationality impacts performance above and beyond common demographic correlates.

Method: We combined published data sets from Argentina, Brazil, Czech Republic, Iran, and the USA resulting in a database of 1,097 healthy adults, before examining the data via multiple regression.

Results: Nationality significantly predicted performance on all three BICAMS tests after controlling for age and years of education. Interactions among the core predictor variables were non-significant. Conclusion: We demonstrated that nationality significantly influences BICAMS performance and established importance of the inclusion of a nationality variable when international norms for the BICAMS are constructed.

Keywords: BICAMS, multiple sclerosis, neuropsychological tests, nationality, demographics 


\section{INTRODUCTION}

A substantial proportion of MS patients experience cognitive impairment. The impairment affects a range of domains, the most frequent being the rate of information processing, visual learning/memory, and to a lesser extent, verbal learning/memory (Benedict et al., 2006). Deficits in these same capacities are related to negative work events in employed patients (Benedict et al., 2016), leading to loss of employment, as well as diminished capacity for activities of daily living and community participation (Goverover, Chiaravalloti, \& DeLuca, 2016). In the 2016 presidential address to the International Neuropsychological Society, Ann Watts PhD cogently argued that neuropsychological services lack accessibility in many countries, particularly those regarded as having low to middle income levels (Boston, USA, February 2016). One obvious impediment to the provision of neuropsychological services is a paucity of reliable and validated assessment tools, with normative data that are relevant to local populations (Shuttleworth-Edwards, 2016).

The Brief International Cognitive Assessment for Multiple Sclerosis (BICAMS) is an effort to address the need for targeted neuropsychological assessment in persons diagnosed with multiple sclerosis (MS) (Benedict et al., 2012; Langdon et al., 2012). BICAMS is a brief battery of three neuropsychological tests that are highly reliable and sensitive to cognitive deficits in MS (Benedict, 2005; Benedict et al., 2006; Benedict et al., 2016; Strober et al., 2009), targeting the key domains referenced above (processing speed, visual learning/memory, and verbal learning/memory). Thus, widespread neuropsychological assessment with the BICAMS can provide clinicians with directly useful information (i.e., current cognitive status) as well as prediction of meaningful outcomes (e.g., vocational function). The battery is intended to provide a cost-effective, easily translated tool, helping clinicians around the world measure neurocognitive impairments and monitor functioning in patients whose cognitive disability may be deteriorating, or transiently impacted by relapses. The BICAMS includes three components: the Symbol Digit Modalities Test (SDMT) oral adaptation to assess cognitive processing 
Smerbeck, et al., Nationality and BICAMS Performance - 4

speed (Smith, 1982), the California Verbal Learning Test, 2nd Edition (CVLT2) learning trials to assess verbal learning and immediate recall (Delis, Kramer, Kaplan, \& Ober, 2000), as well as the Brief Visuospatial Memory Test, Revised (BVMTR) learning trials to assess visuospatial learning and immediate recall (Benedict, 1997).

Since the publication of translation and validation guidelines (Benedict et al., 2012), more than twenty studies have commenced or been completed, spanning five continents (https://www.bicams.net/scientific-background/validation-studies.aspx). This paper is an attempt to determine the impact of nation on BICAMS test results. The nation variable includes a range of influences which are not differentiated in this paper, but which must be understood to ensure that future normative studies include the most relevant demographic variables. Some differences are likely related to language differences among the nations under study. For example, although there are clear translation guidelines (Benedict et al., 2012), the clarity of participant instructions may not be exactly equivalent. Furthermore, the SDMT rewards rapid speech and thus speakers of languages with longer words for numerals may be at a disadvantage. Other factors are potentially more cultural in nature: an abstract shape in the BVMTR becomes easier to retain if it resembles a common road sign or advertising logo prevalent in a particular nation. Thus, the nationality variable is hypothesized to act as a proxy for a broad set of causal factors.

From the outset, our goal has been to provide regression-based norms (RBNs) for the BICAMS so that raw scores are easily transformed to z scores, controlling for demographics deemed instrumental by the clinician user. This paper attempts to widen the scope of the RBNs by examining the inclusion of nationality as a predictor variable. Herein, we address that goal with data from 1097 healthy participants, derived from five national studies (Benedict et al., 2012; Dusankova, Kalincik, Havrdova, \& Benedict, 2012; Eshaghi et al., 2012; Goretti et al., 2014; Spedo et al., 2015; Strober et al., 2009; Vanotti, Smerbeck, Benedict, \& Caceres, 2016). 


\section{METHODS}

\section{Participants}

Participants were 1097 healthy adults recruited from five countries: Argentina, Brazil, Czech Republic, Iran, and United States. All participants were free of substance abuse, psychiatric illness, and any medical problems that could compromise cognitive test performance. All participants reported that their first or primary language was the commonly recognized dominant language for their country of participation: Argentina - Spanish, Brazil - Portuguese, Iran - Persian, Czech Republic - Czech, USA English. All data was collected in compliance with institutional ethical regulations, in compliance with the Helsinki Declaration.

\section{Assessment and Procedures}

This was a retrospective analysis of data obtained from prior MACFIMS/BICAMS validation studies (Dusankova et al., 2012; Eshaghi et al., 2012; Parmenter, Testa, Schretlen, Weinstock-Guttman, \& Benedict, 2010; Spedo et al., 2015; Vanotti et al., 2016). The oral response form of the SDMT, and the learning trials of the CVLT2 and BVMTR were administered in the customary manner, using the translated versions of each test. Thus, the SDMT (Smith, 1982) presents a page with number/symbol pairings displayed in the key at the top of the page. The subject's task was to say the numbers corresponding with each symbol presented in rows below the key, as rapidly as possible. The CVLT2 consists of 16 words which fall in four conceptual categories (Delis et al., 2000). The word list was read five times, each followed by a free recall task. For the purposes of this study, the original English CVLT2 was used in the USA, the Spanish translation of the CVLT was used in Argentina (Artiola I, Hermosillo Romo, Heaton, \& Pardee III, 1998), and the remaining three countries (Brazil, Czech Republic, and Iran) developed their sixteen-item lists by following the published protocol (Benedict et al., 2012). The 
Smerbeck, et al., Nationality and BICAMS Performance - 6

BVMTR is a three-trial task in which the examinee attempts to reproduce an arrangement of six geometric stimuli from memory following a 10-second exposure (Benedict, 1997). Note that the delayed recall trials of the BVMTR and CVLT2 were not required as part of the BICAMS protocol; they were omitted to reduce administration demands.

\section{Statistical Analyses}

Using SPSS v. 21 for Windows, participant age in years and years of education were coded directly. Age² was calculated from the age variable. Sex was dummy coded such that $1=$ male and $2=$ female. Nationality was dummy coded using the Czech Republic as the reference group. Four nationality variables were created (Argentina, Brazil, Iran, and USA) whereby a score of 1 indicates residence in that country and a score of 0 indicates non-residence. Czech nationality was indicated by a rating of 0 in all four countries. Note that while the summary term 'nationality' is used throughout, it is understood that that this variable encompasses a range of influences, including language effects, educational practices, and comparability of test translation. Each of the five nations under study has a different dominant language, so there is no way to differentiate between language effects (such as word length in the SDMT) and cultural effects (such as varying familiarity with CVLT2 objects to be recalled). Thus, while the variable under consideration is referred to as 'nationality', it should be read as referring to all factors which differentiate test performance across countries.

The overall objective was to determine whether nationality yielded meaningful prediction of BICAMS performance above and beyond the predictive effects of a standard set of demographic variables (i.e., age, age ${ }^{2}$, sex, education) established in the prior literature (Parmenter et al., 2010). Age, sex, and education were chosen as standard demographic because they commonly influence neuropsychological test interpretation and indeed are commonly used to stratify neuropsychological test norms. If age-related decline is linear, then $\operatorname{age}^{2}$ will be non-significant. However, if the rate of agerelated decline accelerates through the adult lifespan, as has been found previously (Verhaegen \& 
Salthouse, 1997), the age ${ }^{2}$ variable will allow the model to capture this phenomenon. For each analysis, predictor variables not statistically significant at the $p<0.05$ level were removed from further consideration. (In all cases, raw $p$ values are reported. To control for multiple comparisons, the Bonferroni correction may be applied. In this case, a total of 27 core analyses were conducted, yielding an adjusted $p$-value criterion of .0013 to yield an overall experiment-wise alpha level of .05.)

Furthermore, any variable predicting less than $1 \%$ of the variance in test performance $\left(R^{2} \Delta<.010\right)$ was judged insufficiently clinically significant to retain. The analysis started by assessing the independent predictive utility of each of the four core variables (age, education, sex, and nationality). Second, a hierarchical regression model was used to determine whether the age ${ }^{2}$ variable contributed meaningful influence above and beyond age to determine if a non-linear age model was more appropriate than a linear one. Third, hierarchical regression was used to assess whether nationality was a significant predictor above and beyond the influence of any of the three traditional demographic variables (age, education, and sex) that were retained in the model. Finally, interaction effects were tested. Again, hierarchical regression was used to determine if an interaction contributed meaningful predictive variance above and beyond the simple predictor variables that made up the interaction; for example, whether the interaction between age and education is predictive above and beyond the direct effects of age and education. In all cases of hierarchical regression, the control variable or variables were entered in the first block and the test variable was entered alone in the second block.

\section{RESULTS}

Our sample consisted of 1097 healthy adults, aged $18-65(\mathrm{M}=39.25, \mathrm{SD}=11.63)$, including 343

(31.3\%) men and 754 (68.7\%) women (the proportions reflecting the MS population), with 13.9 (SD = 3.52) mean years of education (Table 1). 
As shown in Table 2, age, education, and nationality were statistically significant and meaningful predictors of all three BICAMS tests, accounting for $4.1-19.1 \%$ of the variance in outcomes $(p<.001$ in all cases). Sex did not significantly predict the $\operatorname{BVMTR}(F(1,1095)=1.807, p=.179)$. Sex was statistically significant for the SDMT $(F(1,1095)=4.770, p=.029)$ and $\operatorname{CVLT2}(F(1,1095)=8.108, p=.004)$, but was not meaningfully predictive of performance (i.e., failed to account for $\geq 1 \%$ of the variance). In addition, sex as a predictor of the SDMT or CVLT2 failed to achieve statistical significance when Bonferroni correction was applied. Since sex did not meet the criteria for meaningful prediction, it was excluded from the remaining core analyses.

A hierarchical analysis found that age $\mathrm{e}^{2}$ contributed unique variance above and beyond the effects of age when predicting SDMT performance $(\mathrm{F} \Delta(1,1094)=18.778, p<.001)$, although the percentage of variance accounted for was small (1.4\%). Age ${ }^{2}$ did not significantly predict CVLT2 performance, once the effects of age were accounted for $(\mathrm{F} \Delta(1,1094)=2.413, p=.121)$. After controlling for age, age ${ }^{2}$ did account for statistically significant variance in BVMTR scores, though not when held to the more stringent Bonferroni-corrected standard $(\mathrm{F} \Delta(1,1094)=7.375, p=.007)$, but failed to meet the $1 \%$ meaningfulness criterion.

Further analysis investigated whether nationality was meaningfully predictive of performance when controlling for basic demographic data - in this case, age and education (since sex was not a substantive predictor, it was dropped from this analysis.) Nationality was a statistically significant predictor of all three BICAMS tests, accounting for $1.5-7.3 \%$ of the variance above and beyond the effects of age and education $\left(\mathrm{F} \Delta_{\text {SDMT }}(4,1090)=31.209, p_{\text {SDMT }}<.001 ; \mathrm{F} \Delta_{\text {CVLT2 }}(4,1090)=5.456, p_{\text {CVLT2 }}\right.$ $\left.<.001 ; \operatorname{F} \Delta_{\text {BVMTR }}(4,1090)=18.515, p_{\text {BVMTR }}<.001\right)$.

The final analysis examined interaction effects between the retained variables. In each case, the interaction was assessed for statistical significance and sufficiently large effect (i.e., explaining at least an additional $1 \%$ of the variance) after controlling for the predictors' main effects. No interaction effect 
accounted for greater than $1 \%$ of the variance in BICAMS scores after controlling for main effects.

Though some $p$-values fell below 0.05 , none met the more stringent Bonferroni-corrected criterion.

Lastly, it should be noted that it is possible for a variable to be part of a significant interaction while not itself being significantly predictive. The variable of sex was dropped from analysis because it was not associated with significant main effects, but there is still a possibility that sex may interact with another variable to produce a meaningful predictor. To examine this possibility, three post-hoc tests were performed to assess whether the interaction between sex and another demographic variable was significant above and beyond the direct effects of the two independent predictors. As seen in Table 2, age-sex and education-sex interactions were non-significant for all dependent variables. The nationality-sex interaction was non-significant for the $\operatorname{BVMTR}\left(\mathrm{F} \Delta_{\mathrm{BVMTR}}(4,1087)=0.845, p=.497\right)$. In the case of the SDMT, the nationality-sex interaction achieved a $p$-value less than.05, but predicted less than $1 \%$ of the variance $\left(\mathrm{F} \Delta_{\mathrm{SDMT}}(4,1087)=2.700, p=.029\right)$. The nationality-sex interaction predicted $1.1 \%$ of the variance in CLVT2 scores above and beyond the individual effects of nationality and sex (F $\Delta$ CVLT2 $\left.(4,1090)=3.084, p_{\text {CVLT2 }}=.015\right)$. At a $p$-value of .015 , this finding is statistically significant on its own, but loses significance when the Bonferroni correction is applied.

\section{DISCUSSION}

This analysis finds robust evidence for the role of age, years of education, and nationality as direct predictors of BICAMS performance, with nationality maintaining predictive power even when age and years of education were held constant. Interactions amongst these variables were not statistically significant or accounted for $<1 \%$ of the variance. Age $^{2}$ predicted a significant, though small, proportion of the variance in SDMT performance after controlling for age, but was not significantly related to either the CVLT2 or BVMTR. On no test did sex account for $\geq 1 \%$ of the variance in BICAMS performance. Posthoc analyses of the interactions between sex and predictor variables were non-significant for the 
BVMTR, and statistically significant but extremely small $\left(<1 \% R^{2} \Delta\right)$ for the SDMT. There was a marginal finding for the interaction between sex and nation in CVLT2 performance only.

Consistent with prior research, participant age and years of education were substantive predictors of all three BICAMS tests. Age predicted approximately $14 \%$ of the variance associated with SDMT and BVMTR performance, while predicting only about $5 \%$ of CVLT2 variance. It should be noted that this data set only extends to age 65 - the model cannot be assumed to extrapolate to older adults. Prior research has shown that processing speed tests are more accurately modeled with both age and age $^{2}$ variables (Verhaegen \& Salthouse, 1997), a finding that was replicated here. Education was similar to age, accounting for about $14-19 \%$ of the variance in BICAMS performance. Past research conducted only within the United States has tended to find greater age than education effects (as judged by standardized $\beta$ weights) (Parmenter et al., 2010); this may be caused by restricted range. That is, in a US-only sample, the range of education outcomes is narrower than in an international sample, thereby restricting the predictive power of the US-sample education variable.

As noted above, influence of sex on performance on processing speed and learning/memory tasks is complex, with findings varying considerably based on task characteristics. Sex was not found to be a meaningful predictor (i.e., statistically significant and accounting for $\geq 1 \%$ of the variance) in this sample for these tasks, nor were interactions between sex and other demographics meaningful, except for a single marginal sex-by-nationality interaction. The ways in which sex influences an individual's set of developmental and educational opportunities probably varies cross-culturally. It is possible that sex, or sex-by-nation interactions, will become more substantive when more national data sets are added to the BICAMS database, potentially increasing the variability. A potential limitation of this study is the skewed participant sex ratio (see Table 1). However, as meaningful sex differences were not found on any of the BICAMS tasks, the high proportion of female participants was unlikely to have any major impact on the results. 
Smerbeck, et al., Nationality and BICAMS Performance - 11

Nationality was a significant predictor of all three tests. As noted above, it should be understood that 'nationality' in this context encompasses a range of factors, including comparability of international BICAMS test forms, and thus the reasons for national differences are likely to be complicated and diverse. Countries are not monolithic in their cultural, linguistic, educational, or political structures - the nationality variable serves only as a gross proxy for any of these factors. Aspects of learning and education may have unique effects in some countries. For example, literacy in a language read left-to-right may confer a modest advantage on a test like the SDMT, which emphasizes left-to-right scanning. Simple linguistic differences, such as syllable length, may confound comparability of word lists on the CVLT2 (Lopez, Steiner, Hardy, Ishak, \& Anderson, 2016). More complicated patterns may emerge as well, such as the relationship between speeded visual processing and the use of a pictographic vs. alphabetic system of writing (Kail, McBride-Chang, Ferrer, Cho, \& Shu, 2013). There is also evidence of neuropsychological scores being influenced by cultural variations, such as differing educational curricula (Janssen \& Geiser, 2011). Clearly, the finding of such differences will vary based on which countries are compared, and may or may not replicate with the nations under consideration in this paper. Thus, while there does appear to be significant between-nation variance that can be accounted for using our RBN methodology, we recognize that future research would be needed to clarify the exact sources of performance differences (i.e., quality of education, distinct linguistic groups within countries, translated test difficulty, etc.) before interpreting the meaning of such findings (Shuttleworth-Edwards, 2016). In particular, comparisons between nations which share a language but differ culturally may help further elucidate the distinction between linguistic and non-linguistic components of the 'nationality' variable. Nonetheless, the nationality variable can be applied without first determining its exact causes. This paper concludes that international applications of the BICAMS (including the establishment of norms) should take nationality into account. A further limitation of this study is the marked sample size differences among national groups. Lastly, while the BICAMS was 
designed to be validly administered alone or as an adjunct to further testing, it remains possible that additional tests given at some sites but not others influenced performance.

This is a first step toward a larger, RBN project for BICAMS with a planned inclusion of 20 or more countries. As the data set grows, other predictors, such as socioeconomic status, MS disease duration, motor factors may be considered, and these factors too will be investigated for interaction effects across nationality. The development of demographically-controlled international BICAMS norms will allow practitioners the flexibility of deciding which interpretive strategy to use. This paper lays the groundwork for the development of such norms by analyzing the properties of a large normative dataset. 


\section{Disclosure of interests}

Author RB is the creator of the Brief Visuospatial Memory Test, Revised, which was used in the present study. 
Smerbeck, et al., Nationality and BICAMS Performance - 14

\section{REFERENCES}

Artiola I, F. L., Hermosillo Romo, D., Heaton, R. K., \& Pardee III, R. E. (1998). Manual de normas y procedimientos para la Bateria Neuropsicologica en Espanol. lowa: Psychology Press.

Benedict, R. H. B. (1997). Brief Visuospatial Memory Test - Revised: Professional Manual. Odessa, Floriday: Psychological Assessment Resources, Inc.

Benedict, R. H. B. (2005). Effects of using same vs. alternate form memory tests in short-interval, repeated assessment in multiple sclerosis. Journal of the International Neuropsychological Society, 11, 727-736.

Benedict, R. H. B., Amato, M. P., Boringa, J., Brochet, B., Foley, F., Fredrikson, S., . . Langdon, D. (2012). Brief International Cognitive Assessment for MS (BICAMS): international standards for validation. BMC Neurol, 12(1), 55. doi:10.1186/1471-2377-12-55

Benedict, R. H. B., Cookfair, D., Gavett, R., Gunther, M., Munschauer, F., Garg, N., \& Weinstock-Guttman, B. (2006). Validity of the Minimal Assessment of Cognitive Function in Multiple Sclerosis (MACFIMS). Journal of the International Neuropsychological Society, 12, 549-558.

Benedict, R. H. B., Drake, A. S., Irwin, L. N., Frndak, S. E., Kunker, K. A., Khan, A. L., . . Weinstock-Guttman, B. (2016). Benchmarks of meaningful impairment on the MSFC and BICAMS. Mult Scler. doi:10.1177/1352458516633517

Delis, D. C., Kramer, J. H., Kaplan, E., \& Ober, B. A. (2000). Califorina Verbal Learning Test - Second Edition. San Antonio, TX: The Psychological Corporation.

Dusankova, J. B., Kalincik, T., Havrdova, E., \& Benedict, R. H. B. (2012). Cross cultural validation of the Minimal Assessment of Cognitive Function in Multiple Sclerosis (MACFIMS) and the Brief International Cognitive Assessment for Multiple Sclerosis (BICAMS). Clin Neuropsychol, 26(7), 1186-1200. doi:10.1080/13854046.2012.725101

Eshaghi, A., Riyahi-Alam, S., Roostaei, T., Haeri, G., Aghsaei, A., Aidi, M. R., . . Sahraian, M. A. (2012). Validity and Reliability of a Persian Translation of the Minimal Assessment of Cognitive Function in Multiple Sclerosis (MACFIMS). Clin Neuropsychol, 26(6), 975-984. doi:10.1080/13854046.2012.694912

Goretti, B., Niccolai, C., Hakiki, B., Sturchio, A., Falautano, M., Eleonora, M., . . Amato, M. (2014). The brief international cognitive assessment for multiple sclerosis (BICAMS): normative values with gender, age and education corrections in the Italian population. BMC Neurol, 14(1), 171. doi:10.1186/s12883-014-0171-6

Goverover, Y., Chiaravalloti, N., \& DeLuca, J. (2016). Brief International Cognitive Assessment for Multiple Sclerosis (BICAMS) and performance of everyday life tasks: Actual Reality. Mult Scler, 22(4), 544-550. doi:10.1177/1352458515593637

Janssen, A. B., \& Geiser, C. (2011). Cross-Cultural Differences in Spatial Abilities and Solution Strategies-- An Investigation in Cambodia and Germany. Journal of Cross-Cultural Psychology, 43(4), 533-557. doi:10.1177/0022022111399646

Kail, R. V., McBride-Chang, C., Ferrer, E., Cho, J. R., \& Shu, H. (2013). Cultural differences in the development of processing speed. Dev Sci, 16(3), 476-483. doi:10.1111/desc.12039

Langdon, D. W., Amato, M. P., Boringa, J., Brochet, B., Foley, F., Fredrikson, S., . . Benedict, R. H. B. (2012). Recommendations for a Brief International Cognitive Assessment for Multiple Sclerosis (BICAMS). Mult Scler, 18(6), 891-898. doi:10.1177/1352458511431076

Lopez, E., Steiner, A. J., Hardy, D. J., Ishak, W. W., \& Anderson, W. B. (2016). Discrepancies between bilinguals' performance on the Spanish and English versions of the WAIS Digit Span task: Cross-cultural implications. Appl Neuropsychol Adult, 23(5), 343-352. doi:10.1080/23279095.2015.1074577

Parmenter, B. A., Testa, S. M., Schretlen, D. J., Weinstock-Guttman, B., \& Benedict, R. H. B. (2010). The utility of regression-based norms in interpreting the minimal assessment of cognitive function in multiple sclerosis (MACFIMS). Journal of the International Neuropsychological Society, 16(1), 6-16. doi:10.1017/S1355617709990750

Shuttleworth-Edwards, A. B. (2016). Generally representative is representative of none: commentary on the pitfalls of IQ test standardization in multicultural settings. Clin Neuropsychol, 30(7), 975-998. doi:10.1080/13854046.2016.1204011

Smith, A. (1982). Symbol digit modalities test: Manual. Los Angeles: Western Psychological Services. 
Spedo, C. T., Frndak, S. E., Marques, V. D., Foss, M. P., Pereira, D. A., Carvalho Lde, F., ... Barreira, A. A. (2015). Cross-cultural Adaptation, Reliability, and Validity of the BICAMS in Brazil. Clin Neuropsychol, 29(6), 836846. doi:10.1080/13854046.2015.1093173

Strober, L., Englert, J., Munschauer, F., Weinstock-Guttman, B., Rao, S., \& Benedict, R. H. B. (2009). Sensitivity of conventional memory tests in multiple sclerosis: comparing the Rao Brief Repeatable Neuropsychological Battery and the Minimal Assessment of Cognitive Function in MS. Mult Scler, 15(9), 1077-1084. doi:10.1177/1352458509106615

Vanotti, S., Smerbeck, A., Benedict, R. H. B., \& Caceres, F. (2016). A new assessment tool for patients with multiple sclerosis from spanish speaking countries: Validation of the Brief International Cognitive Assessment for MS (BICAMS) in Argentina. The Clinical Neuropsychologist, 30(7), 1023-1031. doi:10.1080/13854046.2016.1184317

Verhaegen, P., \& Salthouse, T. A. (1997). Meta-analyses of age-cognition relations in adulthood: Estimates of linear and nonlinear age effects and structural models. Psychological Bulletin, 122(3), 231-249. 
Table 1. Participant characteristics by nation

\begin{tabular}{|l|l|l|l|l|l|c|}
\hline & $\begin{array}{l}\text { Entire } \\
\text { Sample }\end{array}$ & Argentina & Brazil & $\begin{array}{l}\text { Czech } \\
\text { Republic }\end{array}$ & Iran & USA \\
\hline$N$ & 1097 & 150 & 559 & 133 & 89 & 166 \\
\hline Gender & $\begin{array}{c}343 \mathrm{M} \\
754 \mathrm{~F}\end{array}$ & $\begin{array}{l}38 \mathrm{M} \\
112 \mathrm{~F}\end{array}$ & $\begin{array}{c}193 \mathrm{M} \\
366 \mathrm{~F}\end{array}$ & $\begin{array}{c}38 \mathrm{M} \\
95 \mathrm{~F}\end{array}$ & $\begin{array}{c}32 \mathrm{M} \\
57 \mathrm{~F}\end{array}$ & $\begin{array}{l}42 \mathrm{M} \\
124 \mathrm{~F}\end{array}$ \\
\hline Age & $39.3 \pm 11.6$ & $42.8 \pm 10.0$ & $39.3 \pm 12.4$ & $33.5 \pm 8.4$ & $33.8 \pm 9.4$ & $43.3 \pm 10.9$ \\
\hline $\begin{array}{l}\text { Years of } \\
\text { Education }\end{array}$ & $13.9 \pm 3.5$ & $14.9 \pm 2.6$ & $13.0 \pm 4.0$ & $14.3 \pm 2.5$ & $14.3 \pm 3.6$ & $15.3 \pm 2.2$ \\
\hline
\end{tabular}


Table 2. Regression analysis results, variance explained by predictor variables

\begin{tabular}{|l|l|l|l|l|l|l|l|}
\hline Predictor variable & Controlling for & \multicolumn{2}{l}{ SDMT } & \multicolumn{2}{l|}{ CLVT2 } & \multicolumn{2}{l|}{ BVMTR } \\
\hline & & $\mathrm{R}^{2}$ & $\mathrm{p}$ value & $\mathrm{R}^{2}$ & $\mathrm{p}$ value & $\mathrm{R}^{2}$ & $\mathrm{p}$ value \\
\hline Age & & 0.141 & $<.001$ & 0.049 & $<.001$ & 0.143 & $<.001$ \\
\hline Sex & & 0.004 & .029 & 0.007 & .004 & 0.002 & .179 \\
\hline Education & & 0.190 & $<.001$ & 0.191 & $<.001$ & 0.138 & $<.001$ \\
\hline Nationality & & 0.123 & $<.001$ & 0.041 & $<.001$ & 0.083 & $<.001$ \\
\hline & & $\mathrm{R}^{2} \Delta$ & $\mathrm{p}$ value & $\mathrm{R}^{2} \Delta$ & $\mathrm{p}$ value & $\mathrm{R}^{2} \Delta$ & $\mathrm{p}$ value \\
\hline Age ${ }^{2}$ & Age & 0.014 & $<.001$ & 0.002 & .121 & 0.006 & .007 \\
\hline Nationality & Age, Edu. & 0.073 & $<.001$ & 0.015 & $<.001$ & 0.048 & $<.001$ \\
\hline Age-Edu. Interaction & Age, Edu. & 0.001 & .233 & $<0.001$ & .561 & 0.005 & .009 \\
\hline Age-Nat. Interaction & Age, Nat. & 0.007 & .043 & 0.006 & .102 & 0.005 & .142 \\
\hline Edu.-Nat. Interaction & Edu., Nat. & 0.007 & .045 & 0.008 & .031 & 0.009 & .017 \\
\hline & & $\mathrm{R}^{2} \Delta$ & $\mathrm{p}$ value & $\mathrm{R}^{2} \Delta$ & $\mathrm{p}$ value & $\mathrm{R}^{2} \Delta$ & $\mathrm{p}$ value \\
\hline Age-Sex Interaction & Age, Sex & $<0.001$ & .997 & $<0.001$ & .523 & $<0.001$ & .811 \\
\hline Edu-Sex Interaction & Edu., Sex & $<0.001$ & .568 & 0.002 & .121 & 0.001 & .254 \\
\hline Nat.-Sex Interaction & Nat., Sex & .009 & .029 & 0.011 & .015 & 0.009 & .017 \\
\hline
\end{tabular}

Notes:

$N=1097$

SDMT = Symbol Digit Modalities Test; CVLT2 $=$ California Verbal Learning Test, $2^{\text {nd }}$ Edition; BVMTR $=$ Brief Visuospatial Memory Test, Revised; Edu. = Education; Nat. = Nationality

Shaded values indicate meaningful predictors (i.e., statistically significant at the .05 level and accounting for $>1 \%$ of variance).

* When no variables are being controlled for, the total variance explained $\left(R^{2}\right)$ is equal to the variance attributable to the predictor variable $\left(R^{2} \Delta\right)$. 